\title{
Anaesthetic Management of a Patient with Pseudo-TORCH Syndrome
}

\author{
Derya Berk, Alparslan Kuş, Tülay Şahin, Mine Solak, Kamil Toker \\ Department of Anesthesiology and Reanimation, Kocaeli University Faculty of Medicine, Kocaeli, Turkey
}

\begin{abstract}
Background: Pseudo-TORCH syndrome is a rare, chronic disorder that is characterised by dimorphic features such as microcephaly, intracranial calcification, seizures, mental retardation, hepatosplenomegaly and coagulation disorders.

Case Report: We present the anaesthetic management of a forty day-old boy with Pseudo-TORCH syndrome during magnetic resonance imaging. Microcephaly, growth failure, high palate and bilateral rales in the lungs were detected in pre-anaesthetic physical examination. The peripheral oxygen saturation was $88-89 \%$ in room-air and was $95 \%$ in a hood with $5 \mathrm{~L} / \mathrm{min}$ oxygen. We planned general anaesthesia to ensure immobility during magnetic resonance imaging. After standard monitoring, general anaesthesia was induced with $8 \%$ sevoflurane in $100 \% \mathrm{O}_{2}$. After an adequate depth of anaesthesia was reached, we inserted a supraglottic airway device to avoid intubation without the use of a muscle relaxant.
\end{abstract}

Conclusion: In patients with Pseudo-TORCH syndrome, the perioperative anaesthetic risk was increased. We believe that using a supraglottic airway device to secure the airway is less invasive than intubation, and can be performed without the need of muscle relaxants.

Key Words: Pseudo-TORCH syndrome, anaesthesia, supraglottic airway device

Received: 05.05.2012

Accepted: 14.01 .2013

\section{Introduction}

Pseudo-TORCH syndrome is a rare, autosomal recessive, chronic disorder that is characterised by microcephaly, intracranial calcification (ICC), thrombocytopenia, mental retardation, seizures and hepatomegaly (1). Initially, Baraitser et al. (2) defined Pseudo-TORCH syndrome, which is clinically similar to congenital TORCH syndrome caused by toxoplasma gondii, rubella virus, cytomegalovirus and herpes simplex virus. These can only be differentiated between using the negative results of confirmatory tests for TORCH syndrome.

This disease may contribute to intracranial haemorrhage, dysmorphic features, congenital cardiovascular abnormalities, hypo-hyperreflexia and growth failure. To the best of our knowledge, there is no literature about the anaesthetic management of patients with Pseudo-TORCH syndrome.

In this case, we report our anaesthetic management of a forty day-old boy with Pseudo-TORCH syndrome who was complicated by thrombocytopenia, seizures and bilateral pneumonia for magnetic resonance imaging (MRI).

\section{Case Report}

A 23-year old Turkish woman, who had married her uncle's son, was referred to our obstetric clinic in the $37^{\text {th }}$ week of her second pregnancy, following the detection of oligohydramnios on ultrasound imaging. Her first child was a male, who was apparently normal at birth according to the mother, but mental-motor retardation and growth failure was seen after four months.
She was taken for emergency caesarean section immediately and our patient was born premature at 37 weeks of gestational age, with a weight of $2,640 \mathrm{~g}$ (between $5^{\text {th }}$ and $10^{\text {th }}$ percentile), a length of $49 \mathrm{~cm}$ (between $25^{\text {th }}$ and $50^{\text {th }}$ percentile) and a head circumference of $33 \mathrm{~cm}$ (between $5^{\text {th }}$ and $10^{\text {th }}$ percentile). The APGAR score was calculated as 6, 7 and 8 at the first, fifth and tenth minutes of the assessment of physical examination, respectively. Umbilical venous blood $\mathrm{pH}$ was found to be 7.28 by blood gas analysis. He was transferred to the paediatric intensive care unit because of suspected meconium aspiration syndrome. Within the first hours of birth, the boy developed respiratory distress. His peripheral oxygen $\left(\mathrm{O}_{2}\right)$ saturation decreased to $86-87 \%$ and he was taken to hood (Oxyhood, Utah Medical, USA) to receive $8 \mathrm{~L} \mathrm{~min}^{-1}$ oxygen; his saturation raised to $95-96 \%$. He developed generalised tonic-clonic seizures and poorly responded to antiepileptic medication.

Petechiae was noticed on his body. Thrombocytopenia and hyperbilirubinemia were detected. A TORCH screen was sent to the laboratory for PCR; CMV lgG and toxoplasmosis lgG were positive, but herpes lgG and rubella lgG were negative.

Subsequent trans-frontal ultrasound examination showed dilated lateral ventricles and multiple millimetric calcification in the bilateral basal ganglia and periventricular area. Abdominal ultrasound examination showed hepatomegaly. Thoracic $B T$ showed bilateral interstitial pneumonia.

Paediatricians consulted the patient in order to provide sedation during MRI scanning to diagnose intracranial calcifications. The patient was taken to the radiology department 
in the oxygen hood. The patient's pre-anaesthetic physical examination revealed microcephaly, high palate, rough breath sounds, bilateral rales and hepatomegaly. Oxygen saturation was $88-89 \%$ in room-air and $95 \%$ in the hood with $5 \mathrm{~L} / \mathrm{min} \mathrm{O}_{2}$. His blood count results were as follows: $\mathrm{Hb}: 11.1 \mathrm{~g} / \mathrm{dL}, \mathrm{Hct}$ : 31.4\%, platelet: 35. 4x 103/UL, pt: $11.8 \mathrm{sec}$, INR: 0.91, SGOT: $113 \mathrm{U} / \mathrm{L}, \mathrm{SGPT}: 135 \mathrm{U} / \mathrm{L}$. Arterial blood gas analyses were: $\mathrm{PO}_{2}$ : $78.8 \mathrm{mmHg}, \mathrm{PCO}_{2}: 49.8 \mathrm{mmHg}, \mathrm{pH}: 7.32, \mathrm{SO} 2: 95.6 \%$, lactat: $12 \mathrm{mg} / \mathrm{dL}, \mathrm{Hb}: 11.8 \mathrm{~g} / \mathrm{dL}, \mathrm{Hct}: 35.4 \%, \mathrm{HCO}_{3}: 25.5 \mathrm{mmol} / \mathrm{L}$.

In the MRI department, after informed patient consent, standard monitoring was performed: heart rate: 150 beats/ $\mathrm{min}$, respiratory rate: 25 breath/min, non-invasive blood pressure: $86 / 48 \mathrm{mmHg}$. The patient was pre-oxygenated with $100 \%$ oxygen. General anaesthesia was induced with $8 \%$ sevoflurane in $100 \%$ oxygen. Face-mask ventilation was easy. Proseal-LMA (PLMA, Laryngeal Mask Company, United Kingdom) was inserted for airway management.

Anaesthesia was maintained with $2.5 \%$ sevoflurane in $50 \%$ oxygen in air. Pressure-controlled ventilation was initiated and respiratory parameters were monitored constantly with an MRI-compatible anaesthetic machine (Datex-Ohmeda, S/5, GE Healthcare, Helsinki, Finland).

The MRI took 45 minutes. Sevoflurane was discontinued and the fresh gas flow increased to $6 \mathrm{~L} / \mathrm{min} \mathrm{O}_{2} 100 \%$ to wash out volatile anaesthetics. After removal of the PLMA, the patient was transferred to the paediatric intensive care unit with hood at a rate of $6 \mathrm{~L} / \mathrm{min}$. No complications, such as bradycardia or severe desaturation $\left(\mathrm{SpO}_{2}<90\right)$, were detected during the whole procedure.

MRI reports showed a parenchymal haematoma sized 8 $\mathrm{mm}$ in diameter in the left thalamus and bilateral focal haemorrhagic and calcific areas in the lateral ventricles.

\section{Discussion}

The patient was revealed to have microcephaly, hepatomegaly, thrombocytopenia, jaundice, seizures, ICC and dilated lateral ventricles on MRI scan; serological tests were negative. The first diagnostic impression for this case is Pseudo-TORCH syndrome. Twenty-seven patients with Pseudo-TORCH syndrome were reported in the literature (2-3).

General anaesthesia is relatively safe for children who receive anaesthesia outside the operating room, for example during MRI scanning. Different alternative approaches have been published regarding the anaesthesia management for preventing movement of the patients (4).

During general anaesthesia, endotracheal intubation is the gold standard to secure the airway. Intubation with laryngoscope might be difficult with anatomic defects such as microcephaly and high palate and abnormal bleeding parameters. When difficult intubation was expected, supraglottic airway devices may be used to reduce haemodynamic changes caused by laryngoscopy. One of the most commonly used SGA is the laryngeal mask airway PLMA. We chose PLMA for ease of positioning, reduced invasiveness and preventing mucosal trauma (bleeding) (5). In the literature, the first attempt success rates for PLMA were reported to range between $76 \%$ and $100 \%(6,7)$. In a report of patients receiving general anaesthesia, the use of the supraglottic airway device resulted in a statistically lower incidence of complications such as laryngospasm and coughing compared to endotracheal intubation (8).

In this case report, we planned general anaesthesia to guarantee the patient's airway. We used sevoflurane for the induction and maintenance of anaesthesia to prevent straining. Sevoflurane is an excellent choice due to its ability to rapidly increase alveolar concentration and its pleasant smell, making it a calm and quick inhalation induction method for paediatric patients.

In conclusion, when a child presents with microcephaly, ICC and petechiae, the physician should consider PseudoTORCH syndrome. Patients with this syndrome often experience abnormal coagulation parameters, dimorphic features and seizures; these will increase the perioperative anaesthetic risk. We recommend the use of supraglottic airway devices because of haemodynamic stability, a reduced risk of bleeding and reduced need for muscle relaxants in patients with Pseudo-TORCH syndrome.

\section{Ethics Committee Approval: N/A.}

Informed Consent: Written informed consent was obtained from the patients parent.

Peer-review: Externally peer-reviewed.

Author contributions: Concept - D.B., A.K.; Data Collection\&/or Processing - D.B., A.K.; Analysis\&/or Interpretation - D.B., A.K., T.Ş.; Literature Search - D.B.; Writing - D.B.; Critical Reviews - M.S., K.T.

Conflict of Interest: No conflict of interest was declared by the authors.

Financial Disclosure: No financial disclosure was declared by the authors.

\section{References}

1. Abdel-Salam GHM, Zaki MS, Saleem SN, Gaber KR. Microcephaly, malformation of brain development and intracranial calcification in sibs: pseudo-TORCH or a new syndrome. Am J Med Genet Part 2008;146:2929-36. [CrossRef]

2. Aicardi J, Goutières F. A progressive familial encephalopathy in infancy, with calcification of the basal ganglia and chronic cerebrospinal fluid lymphocytosis. Ann Neurol 1984;15:49-54. [CrossRef]

3. Knoblauch H, Tennstedt C, Brueck W, Hammer H, Vulliamy T, Dokal I, et al. Two brothers with findings resembling congenital intrauterine infection-like syndrome (Pseudo-TORCH Syndrome). Am J Med Genet Part 2003;120:261-5. [CrossRef]

4. Cauldwell $C$. Anesthesia risks associated with pediatric imaging. Pediatr Radiol 2011;41: 949-50. [CrossRef]

5. Trevisanuto D, Grazzina N, Ferrarese P, Micaglio M, Verghese C, Zanardo V. Laryngeal mask airway used as a delivery conduit for the administration of surfactant to preterm infants with respiratory distress syndrome. Biol Neonate 2005;87:217-20. [CrossRef]

6. Gaitini LA, Vaida SJ, Somri M, Yanovski B, Ben-David B, Hagberg CA. A randomized controlled trial comparing the ProSeal laryngeal mask airway with the laryngeal tube suction in mechanically ventilated patients. Anesthesiology 2004;101:316-20. [CrossRef]

7. Brain Al, Verghese C, Strube PJ. The LMA ,ProSeal'-a laryngeal mask with an oesophageal vent. Br J Anaesth 2000;84:650-4. [CrossRef]

8. Yu SH, Beirne OR. Laryngeal mask airways have a lower risk of airway complications compared with endotracheal intubation: a systematic review. J Oral Maxillofac Surg 2010;68:2359-76. [CrossRef] 